\title{
An Adaptive Guidance System for Robotic Walking Aids
}

\author{
Birgit Graf \\ Fraunhofer Institute Manufacturing Engineering and Automation (IPA), Stuttgart, Germany
}

In the last years, several robotic walking aids to assist elderly users with mobility constraints and thus to react to the growing number of elderly persons in our society have been developed. In order to ensure good support for the user, the robotic walker should adapt to the motion of the user while at the same time not losing the target out of sight. Even though some of the existing active robotic walkers are able to guide their user to a target, during guidance, the input of the user is not considered sufficiently. Therefore a new adaptive guidance system for robotic walkers has been developed. It is able to lead the walking aid user to a given target while considering his inputs during guidance and adapting the path respectively. The guidance system has been implemented on the mobile robot assistant Care-O-bot II and a field test was done in an old people's residence proving the correct function and usefulness of the guidance system.

Keywords: walking aid, guidance, path planning, path modification, shared control, service robot

\section{Introduction}

In the last years, the percentage of elderly people in our society has grown rapidly. Out of 82.5 million people living in Germany in 2005, according to numbers of the German Federal Statistical Office, around 19 percent were seniors above 65 years [26]. With the demographic development continuing, in the year 2050 the number of people above 65 years will comprise between 33 and 36 percent of Germany's population. Similar numbers are reported from other industrial nations all over the world, in particular the United States and Japan. Many elderly people suffer from mobility constraints and thus depend on the use of mobility aids such as wheelchairs, walkers, walking canes or crutches. According to a study conducted in the USA in 2000, 14 percent of all persons above
65 years use some kind of mobility aid and 64 percent of all mobility aids are used by over 65year-olds [13]. Even when using a mobility aid, many elderly people need to be accompanied by nurses when moving around. Other people use wheelchairs even though they are still able to walk. Reasons are the high risk of falls caused by unstable walking or by muscle weakness, the risk of collisions caused by cognitive disabilities, or mental disorders preventing elderly persons to find their way around their environment. According to a study conducted in the US between 1987 and 1992, over this six year period, an average of one percent of all walking aid users had an accident which involved walker use and which required emergency room treatment [2].

Robotic walkers able to perceive and localize themselves in their environment and to guide their user to a target support and enhance the independence of elderly or disabled persons with mobility constraints. In order to ensure good support for the user, the robotic walker should adapt to his motion while at the same time not losing the target out of sight. The following Section gives a summary of existing robotic walking aids and their capabilities and shared control systems of robotic wheelchairs. Some of the existing active systems are able to guide their user to a target. However, during guidance, the input of the user is not considered sufficiently. Therefore, in Section 3, a new adaptive guidance system for robotic walkers is presented. The system is able to lead the walking aid user to a given target while considering his inputs and thus ensuring that the user of the robotic walker feels in control of the machine and gets the necessary support at all time. The guidance 
system has been implemented on the mobile robot assistant Care-O-bot II which can also be used as a walking aid. It has been tested with elderly users, the results of the field tests will be reported in Section 4. Section 5 concludes with an outlook on future works.

\section{State of the Art}

In the last years, several research facilities have enhanced off-the-shelf walkers by integrating robot technology such as environment sensors and drives. New walking aid robots with individual design and mechanics provide additional support to their user, ranging from audio or visual information on the environment to autonomous or semi autonomous navigation. For existing robotic walkers, two basic types can be distinguished:

Passive robotic walkers do not have any driven wheels and move directly according to the applied user forces (direct user control). Examples for passive walkers are the COOL Aid [11] and PAMM-AID [14] system, which use motorized steering of the wheels to lead the user around obstacles. The RT-Walker [9] uses a special servo brakes to steer the device in a collision-free direction. In case no obstacles are detected the user has full control on the device and can control it similarly to a conventional walker. Most passive systems are equipped with brakes to stop if they are getting too close to an obstacle or step. A guidance system for Guido, the commercial successor of PAMM-AID has been presented in [23]. After a target has been set, the robot will plan and follow a path to the target. During guidance the desired direction indicated by the user input will be ignored. The successor of the RT-Walker, ORTW-II [20] uses a "potential canal" method which only allows deviations up to a certain distance from the optimal path to the target. The CMU roboticallyaugmented walker [6] does not use its motors when traveling with a user but tracks its position and displays the optimal direction of travel on a screen to guide its user to the selected target.

Active robotic walkers are equipped with motorized wheels. Force or force-torque sensors of different kind are used to determine the forces applied to the system by the user. These forces are used to calculate the desired driving direction and speed of the walker (indirect user control). Some systems (Silbo [25], Hitachi walker [5], RT-walker) are equipped with angle sensors enabling to adapt the required force input at slopes. All systems are able to detect obstacles and stop in front of them; most of them adapt the traveling direction to surround obstacles in advance. The PAMM smart walker [28] and the CMU robotic walker [19] are able to localize themselves in their environment and thus plan an optimal path to a given target. The shared control system of PAMM creates a virtual force leading the robot to the given target that is combined with the real forces applied to the device by the user. The gains of each control input are set depending on the observed user abilities. Whereas for PAMM, an interface providing feedback to the user on the planned path has not been presented, the CMU robotic walker displays the desired direction of travel on a screen. The user is free to move in any direction; however, if the deviation to the planned path is too large, the velocity of the walker will be reduced to force the user back to the path.

Other robotic walkers focus their works on additional support functions such as lifting assistance (Hitachi walker, Monimad walker [18], MOBIL Walking \& Lifting Aid [1], Walking helper II [3]), person tracking (MOBIL Test Bed [24]), or moving out of the way when not used (CMU robotically-augmented walker). The walking support system REHABOT, presented in [16], uses a robotic manipulator, a mobile platform, a harness, and sensor system to provide safe support for the elderly or the disabled in rehabilitation programs.

Different shared control systems have been presented for robotic wheelchairs. In most systems the user is responsible to steer the device to a target whereas local modifications of the desired direction are applied by the computer controls. Existing control systems can be divided into two types [4]: model-based approaches such as the MVFH [17] that generates a histogram of the environment identifying the optimal direction of travel, or behavior-based approaches that activate specific local behaviors such as obstacle avoidance, docking, wall following etc. MAid [21] is a robotic wheelchair able to provide autonomous guidance functions. However, it does not consider any user inputs during guidance. 


\section{Implementation}

The adaptive guidance system provides two basic operation modes [7]: "Direct Control Mode" enables the user to push or pull the robotic walker to a certain direction, whereas the velocity of the robot is reduced in front of obstacles or when a stumble motion of the user has been detected. In "Target Mode" the user follows the robotic walker along a pre-planned, optimal path to a previously specified target. In this mode, the velocity of the walker is calculated from the user input as in the direct control mode, the direction of motion, however, is given by the planned path. The path is modified by detected obstacles or user input. The single modules of the navigation system implementing the adaptive guidance system are displayed in Figure 1.

Some components of the control system, e.g. for self localization, motion control or collision avoidance, are the same for robotic walkers and other mobile service robots and will therefore not be discussed in this publication. The motion generator module is specific for the walking aid functionality. It is used to calculate the desired motion direction and velocity from the forces applied to the robotic walker by the user. In direct control mode, the output of this module is directly used to calculate the desired velocity and motion direction of the robot. The path planning and path modification modules and the behavior selection module are only used in target mode. The latter evaluates the desired motion direction of the user provided by the motion generator and adapts the path to the target accordingly. These three modules of the guidance system will now be described in detail.

\subsection{Motion Generator}

The motion generator analyses the applied user forces and torques to determine the velocity and motion direction desired by the user. The motion generation consists of two steps:

1. Stumble detection. The forces applied to the walker are compared to previous sensor readings and checked for large differences. This indicates that the user may have stumbled and should stop the robot instead of accelerating it. A force difference of $50 \mathrm{~N}$ within 0.2 seconds has been identified as a good value for stumble detection. In case a potential stumble of the walking aid user has been detected, the user forces are reduced to zero. The stumble protection will stay activated until the applied user forces have become zero or are applied in the different direction. This ensures that the user has regained his balance and the walker can safely move on.

2. Calculation of desired velocities. The acceleration of an off-the-shelf walker is directly derived from the applied user forces. When not pushed, the walker is slowed down by the friction of its wheels on the ground. The mass model implemented in the motion generator is based on the motion behavior of conventional

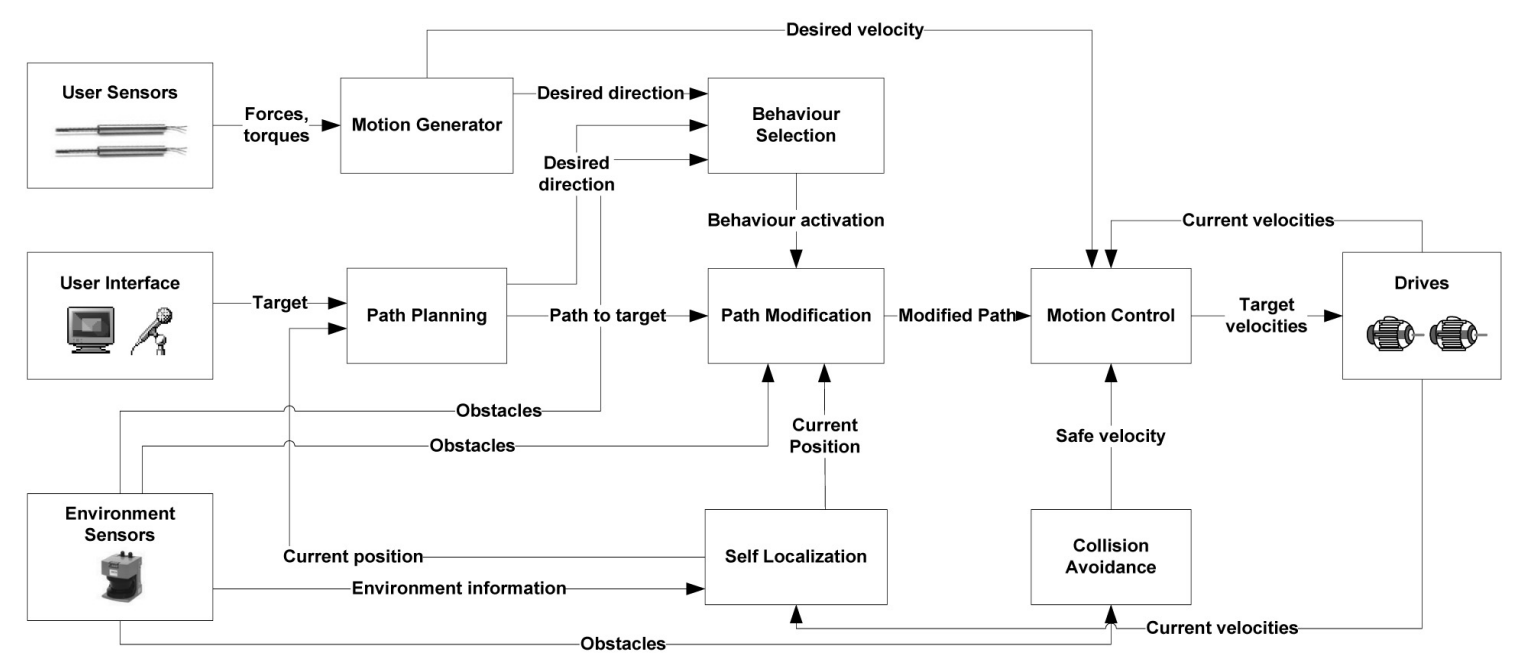

Figure 1. Components and data flow of the guidance system. 
walkers. Depending on the maximum allowed forces and acceleration, the target velocity of the walker caused by the user input alone is calculated as

$$
v_{U s e r, t}=v_{0}+\int \frac{a_{\max } \cdot F_{U s e r}(t)}{F_{\max }} d t .
$$

The simulated friction opposing this velocity is calculated as

$$
v_{\text {Roll }, t}=\int a_{\text {Roll }}(t) d t
$$

where

$$
\mathrm{a}_{\text {Roll }}=\frac{\mathrm{F}_{\text {Roll }}}{\mathrm{m}}, \quad F_{\text {Roll }}=m \cdot g \cdot c_{r} .
$$

As the friction always acts in the direction opposed to the current motion direction, the final velocity $v_{V e h, t}$ of the walker is calculated as:

$$
\begin{array}{rll}
v_{\text {Veh }, t}=0 ; & \text { for } & \left|v_{\text {User }}\right| \leq v_{\text {Roll }} \\
v_{\text {Veh }, t}=v_{\text {User }}-v_{\text {Roll }} ; & \text { for } & \left|v_{\text {User }}\right|>v_{\text {Roll }} \\
\text { und } \quad v_{\text {User }}>0 & & \\
v_{\text {Veh }, t}=v_{\text {User }}+v_{\text {Roll }} ; & \text { for } & \left|v_{\text {User }}\right|>v_{\text {Roll }} \\
\text { und } \quad v_{\text {User }}<0 . & &
\end{array}
$$

The maximum allowed user forces for the robotic walker has been restrained to $40 \mathrm{~N}$ which is twice the maximum force applied to a conventional walker. The control parameters $a_{\max }$ and $c_{r}$ of the above equation were determined by the following tests:

- Accelerate walker to maximum velocity, let go of walker handles and measure time to stop.

- Accelerate walker to maximum velocity, stop walker manually by pulling handles, measure time to stop.

- Move walker with constant velocity.

The $c_{r}$ value measured for conventional walkers $(0.02)$ in previous evaluations proved not to be applicable for the robotic walker. Finally $\mathrm{a}_{\max }=3 \mathrm{~m} / \mathrm{s}^{2}$ and $c_{r} \cdot g=0.5$ were determined as the optimum parameter settings allowing the robotic walker to stop fast enough to provide sufficient support to the user while at the same time reducing the forces required to start the walker and to maintain a constant velocity to an acceptable value $(<7 \mathrm{~N})$.

\subsection{Path Planning}

Global path planning is based on an environment map containing all static obstacles in the environment and calculates an optimal path from the current position to a given target. Two different approaches for global planning have been implemented and can be selected according to the abilities of the user, the structure of the environment and the geometry of the robot.

A grid-based potential field planner based on "wavefront expansion" [15] is applied for a robot allowed to turn on the spot and without restrictions of the turning radius. Obstacles are grown by the robot collision radius and given a high potential. After the target has been specified, the Dijkstra algorithm is applied to set the potential of each grid according to the costs to get to the target. Figure 2 shows an example of a path planned in a 2-dimensional configuration space. Figure 3 displays the $3-\mathrm{D}$ view of the potential field with falling potential from start to goal. The path is determined by performing a steepest gradient descent on the potential function.

The non-holonomic planner is based on [12] and considers a limited turning radius of the robot and avoids tuning on the spot. It calculates the reduced visibility graph (RVVG) for a robot at the shape of a point. In the RVVG, the shortest path is obtained using the $\mathrm{A}^{*}$ algorithm. In a second step this shortest path is evaluated whether it could be modified to suit the kinematic and geometric constraints of the

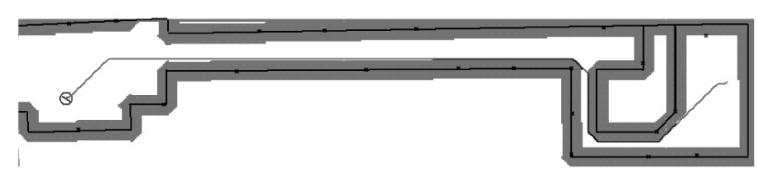

Figure 2. Path generated by a potential field planner.

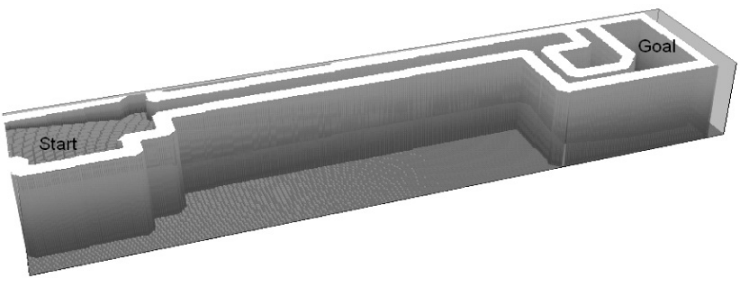

Figure 3. 3-D visualization of potential field used to calculate path in Figure 2. 
robot. The search continues until a feasible path has been found which is then adapted to suit the robot. Figure 4 displays a suitable path for a robot not allowed to rotate on the spot and with limited turning radius.

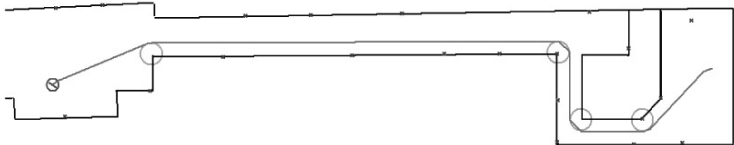

Figure 4. Non-holonomic planning with a maximum turning angle of $50 \mathrm{deg}$.

\subsection{Path Modification}

The method of elastic bands [22] is used for local path modifications, specifically to smoothen the path returned by the global planner, to avoid dynamic obstacles, and to consider the input of the walking aid user. An elastic band is a deformable collision free path. It is represented as a sequence of connected "bubbles". The bubbles are created by examining the local free space of the robot at configurations along this path. A bubble $B(q, r)$ of an elastic band represents all configurations $p$, which the robot can reach from its current configuration $q$ without collision. The radius $r$ of a bubble is defined as the minimum distance to the closest obstacle (Figure 5).

$$
\begin{aligned}
B(q, r) & =\left\{p \in \mathcal{R}^{\mathrm{N}}:\|q-p\|\right. \\
& \left.<r(q) \wedge|q-p| \geq r_{\text {veh }, \text { min }}\right\}
\end{aligned}
$$
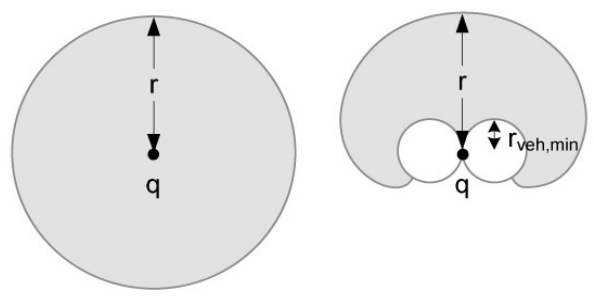

Figure 5. Bubbles for a robot without limitations and a Dubin's car with limited turning radius $r_{r o b, m i n}$.

The elastic band is applied to the path returned by the global planner. In order to optimize the bubble band, each bubble is continuously modified by external and internal forces. Internal forces $F_{i}$ pull each bubble towards its neighbors and thus remove any possible slack in the path (Figure 6).

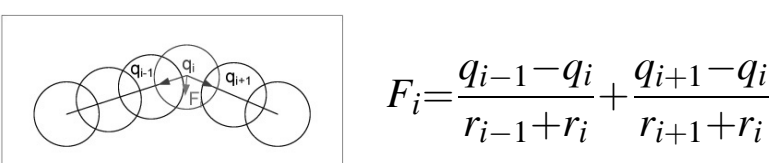

Figure 6. Internal forces between bubbles.

External forces $F_{e}$ push the path away from obstacles (Figure 7). For all bubbles within the influence distance $o$ to an obstacle, a rejecting force at the size of the potential $\phi(q)$ is applied that increases the smaller the distance $d$ of the bubble $q$ to the closest obstacle point $c l p$ :

$$
\begin{array}{r}
F_{e}=\sum_{c l p:|q-c l p|<o} \phi(q) \cdot(q-c l p), \\
\phi(q)=\tan \left(\frac{\pi}{2}\left(1-\frac{d}{o}\right)\right)
\end{array}
$$

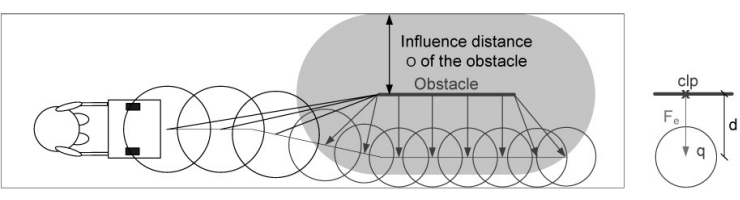

Figure 7. External forces applied to selected bubbles caused by an obstacle.

Figure 8 and Figure 9 display the optimized elastic bands for a holonomic robot and a Dubin's car with limited turning angle. The detailed non-holonomic path initialization is described in our previous work [10].

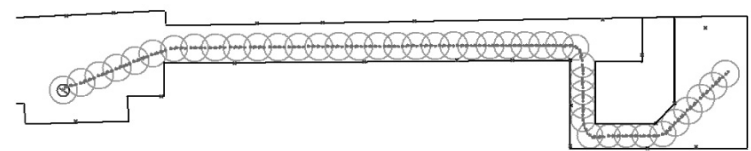

Figure 8. Elastic band covering the path in Figure 2 for a holonomic robot.

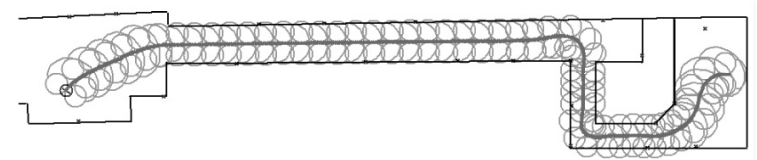

Figure 9. Elastic band covering the path in Figure 4 for a robot with a maximum turning angle of $50 \mathrm{deg}$. 
In order to adapt the path to the desired direction of the user, additional modifications of the elastic band can be activated. Direct user forces (Figure 10) move all the bubbles towards the desired motion direction dir of the user:

$$
F_{d}=\operatorname{dir}
$$

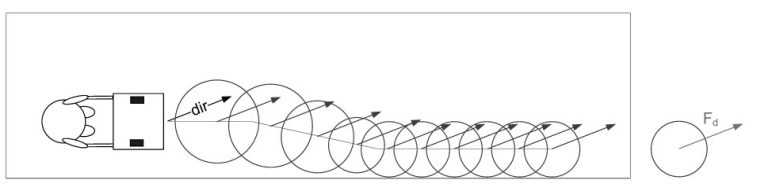

Figure 10. User forces applied to all bubbles of the band.

Behavior forces $F_{b}$ enable the activation of docking or wall following behaviors on the way to the target. Docking behavior is important in case the user wants to pick something up on the way to the target or stops to talk to a friend. Wall following can be used to avoid other people in a corridor and to stabilize the motion of the walker. The activation of behavior forces provides one important advantage compared to the direct path modification described previously. As the robot maintains a behavior until other conflicting direction commands are given, the required forces for adapting the path to user inputs can be reduced to a short period.

For docking (Figure 11), the elastic path will be modified to lead the user as close as possible to the designated docking point. Therefore all bubbles in the vicinity are drawn towards the intermediate target $\mathrm{Z}$ :

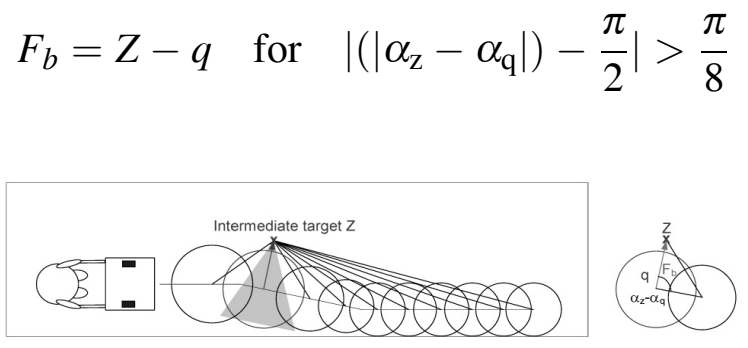

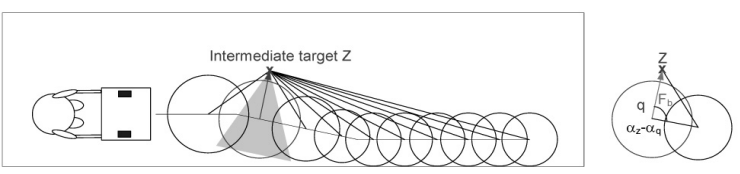

Figure 11. Behavior forces applied to selected bubbles for docking to an intermediate target.

For wall following (Figure 12), all bubbles close to the wall segment $w$ are drawn towards it:

$$
F_{b}=c l p-q \text { for }\left|\alpha_{\mathrm{clp}}-\alpha_{\mathrm{w}}\right|=\frac{\pi}{2}
$$

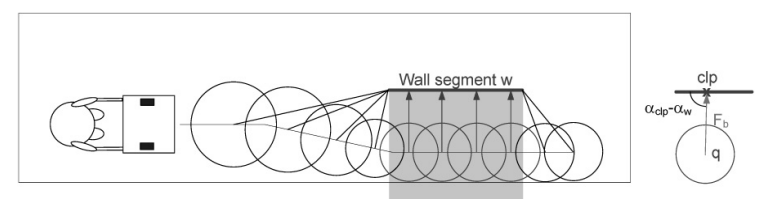

Figure 12. Behavior forces applied to selected bubbles for wall following.

The position of each bubble located within a certain distance to the robot is modified according to the applied forces. The resulting force applied to each bubble is the weighted sum of all applied forces:

$$
\begin{aligned}
& \operatorname{Pos}_{\text {new }}=\operatorname{Pos}_{\text {old }}+\delta \cdot F_{\text {res }} \\
& F_{r e s}=\mathrm{k}_{\mathrm{i}} \cdot F_{i}+\mathrm{k}_{\mathrm{e}} \cdot F_{e}+\mathrm{k}_{\mathrm{d}} \cdot F_{d}+\mathrm{k}_{\mathrm{b}} \cdot F_{b} .
\end{aligned}
$$

For collision-free motion, the external forces created by obstacles must always be larger than the forces created by user input. A stable motion behavior could be reached for $k_{i}=0.25, k_{e}$ $=0.2, k_{d}=0.05, k_{b}=0.1$.

\subsection{Behavior Selection}

This module combines the input of the user with the result from the path planner and activates the specific path modifications of the elastic band described in 3.3. Depending on the nature of the currently planned motion, i.e. rotation or forward motion, the user-desired linear velocity and motion direction are compared to the planned motion direction (Figure 13). In case of large differences, i.e. wrong rotation direction or backwards motion desired, we assume that the user has changed plans and does not

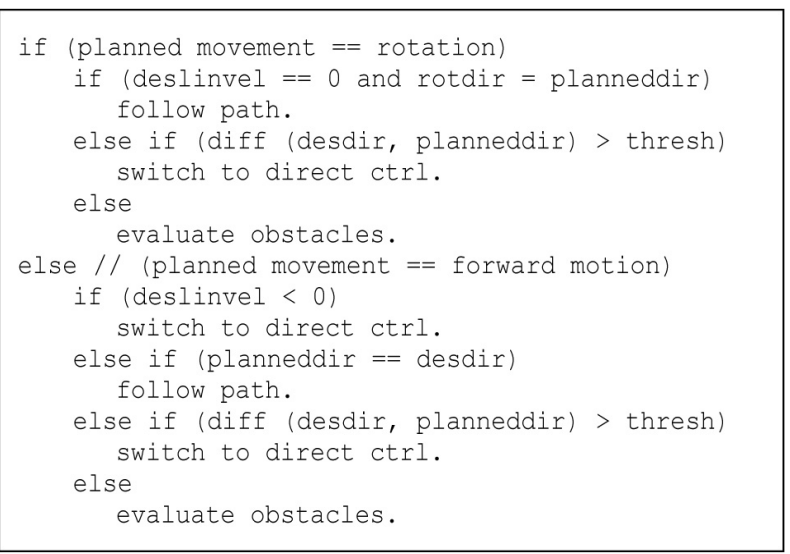

Figure 13. User input evaluation algorithm based on planned motion direction (planneddir) and user desired linear velocity (deslinvel), and direction (desdir). 
want to proceed to the target any more. Therefore, the operation mode will switch to direct control and the robotic walker will not move on to the target. For small differences the robot follows the planned path or starts the obstacle evaluation to determine suitable path modifications.

If differences between the desired and the planned motion direction are observed for some time, the environment will be examined for obstacles and an appropriate path modification strategy will be selected (Figure 14).

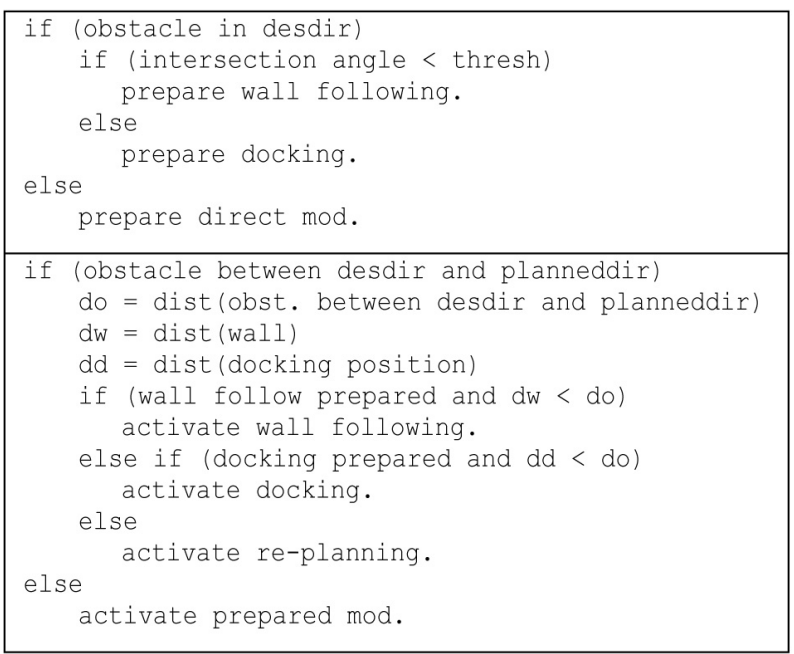

Figure 14. Obstacle evaluation algorithm based on detected obstacles in desdir (intersecting obstacles) and between desdir and planneddir (not intersecting obstacles).

For wall following, a wall segment located in the desired direction, for docking an intermediate target at a certain distance to the selected docking object will be calculated (Figure 15). If the user pushes towards the other side of an obstacle than initially planned, it may also become necessary to do a global re-planning of the path. The new path will lead the user trough

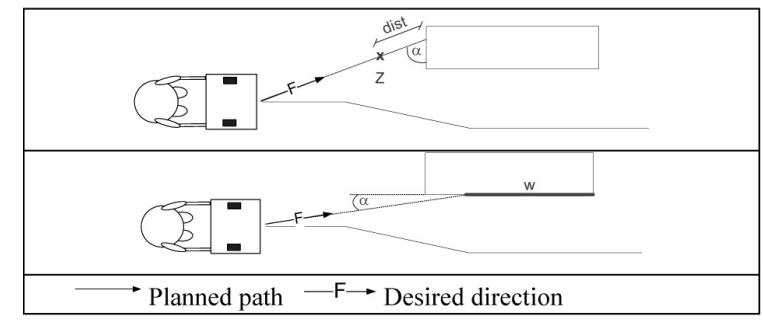

Figure 15. Parameters for docking and wall following: wall $w$, target $Z$.

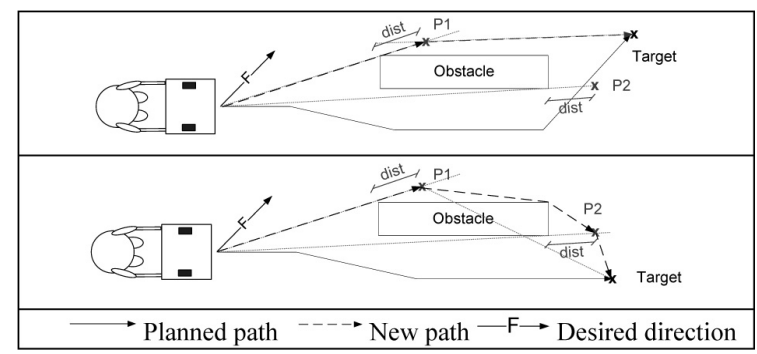

Figure 16. Parameters to drive around an obstacle on the other side.

intermediate path positions $P 1$ and $P 2$ on the desired side of the obstacle (Figure 16).

In order to determine an appropriate path modification, the closest obstacle intersecting the desired motion direction of the user is determined as a potential docking target or wall to follow. Additionally, the closest obstacle between the desired motion direction and the planned direction is calculated. To determine whether to activate docking or wall following or to re-plan around the other side of an obstacle, the distances to each obstacle are compared. Figure 17 shows two examples where both kinds of obstacles are available.

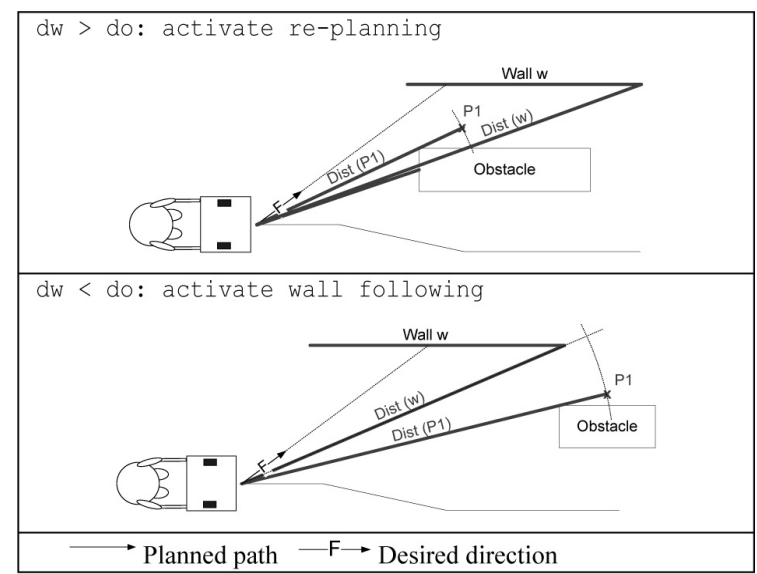

Figure 17. Decision between wall following or re-planning.

\section{Field Tests}

\subsection{Care-O-bot II}

Care-O-bot II (Figure 18) is the second prototype of a mobile robot assistant designed to assist elderly people in home environments. It is equipped with a manipulator arm, adjustable 


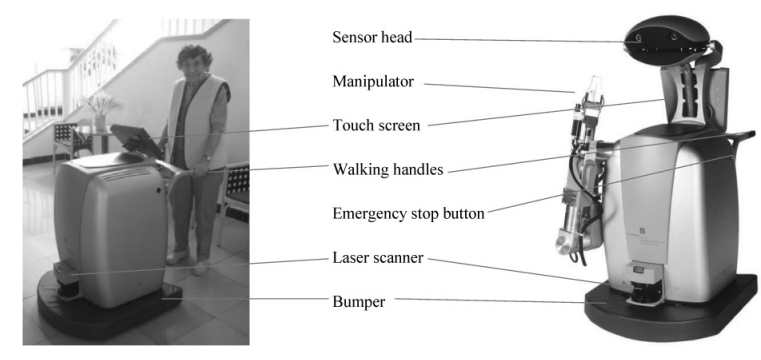

Figure 18. Care-O-bot II without sensor head and manipulator (left) and fully assembled system (right).

walking supporters, a tilting sensor head, and controlled by a hand-held control panel.

Care-O-bot II consists of two autonomous systems: the mobile platform with the walking supporters and the top level with the manipulator arm and sensor head. Both systems can be disconnected easily as they both work independently with their own PC, power supply, and emergency control circuit. The top level platform is not required for the functionality of the robot as a walking aid and will therefore not be discussed any further. For an overview of all capabilities of Care-O-bot II, please see [8].

For environment perception, Care-O-bot II is equipped with a laser scanner. A bumper and two emergency buttons are used as an additional safety system to prevent collisions. The walking aid handles of Care-O-bot II can be adjusted in height and are equipped with force sensors to measure the forces applied to each handle. The force and torque acting in the centre of the robot are calculated from the forces applied to the handles.

Previous user evaluations revealed that most users had difficulties to operate the robotic walker with the top level system as it blocked their view along the path. Therefore, for the evaluation of the guidance system, the top level system was taken off the robot and a separate touch screen attached. The touch screen interface to control the robot during the user tests is displayed in Figure 19. In addition to the status

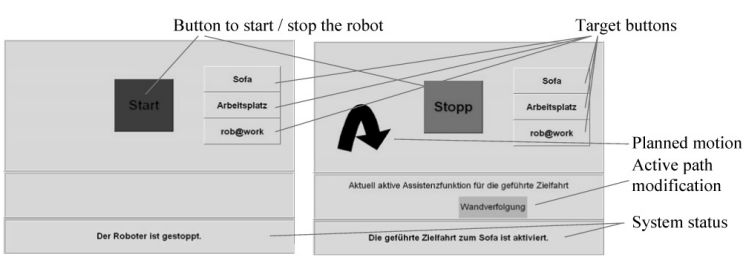

Figure 19. User interface for field trials. messages on the screen, audio messages were used to inform the user on the current target and operation mode.

\subsection{Test Environment and Persons}

The latest user tests were done in an old people's residence in Stuttgart-Berg. The residence consists of three buildings connected by corridors.

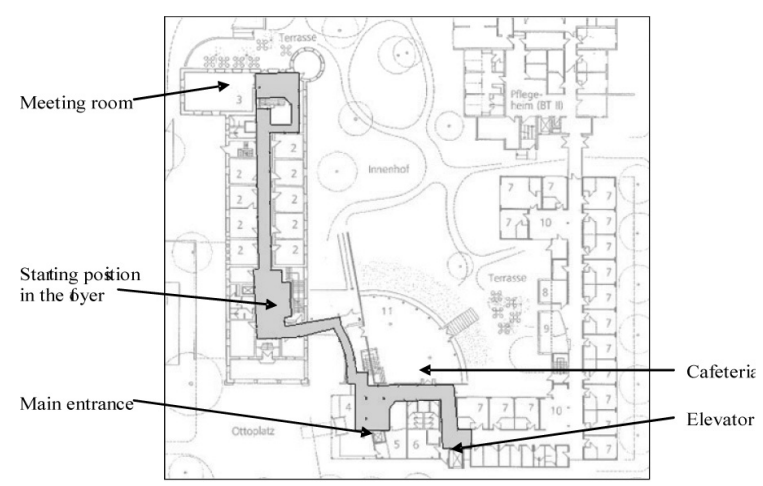

Figure 20. Layout of the ground floor and corresponding robot map (grey area visualizes operation area).

Representative target positions in two of these buildings and in two different floors were selected for the evaluation of the guidance system. The size of the navigation area on the ground floor was around $40 \times 70 \mathrm{~m}$, in the first floor around $10 \times 20 \mathrm{~m}$. Six inhabitants of the residence between 86 and 92 years, 5 female, 1 male were selected for the tests, each of them using mobility aids in their daily life.

\subsection{Comparison of Walkers}

For the comparison between conventional and robotic walker, the test persons were asked to walk from a starting position in the foyer to the target in front of the meeting room and back. Chairs were positioned in both target positions for the user to rest between trials. The path led from a large hall along a corridor and down a ramp. This test was first done with a conventional walker equipped with a bicycle speedometer to measure walking distance and duration. After that, the use of the robotic walker was explained to the test persons and the same test was done twice with the robotic walker, first in direct control mode, then in 

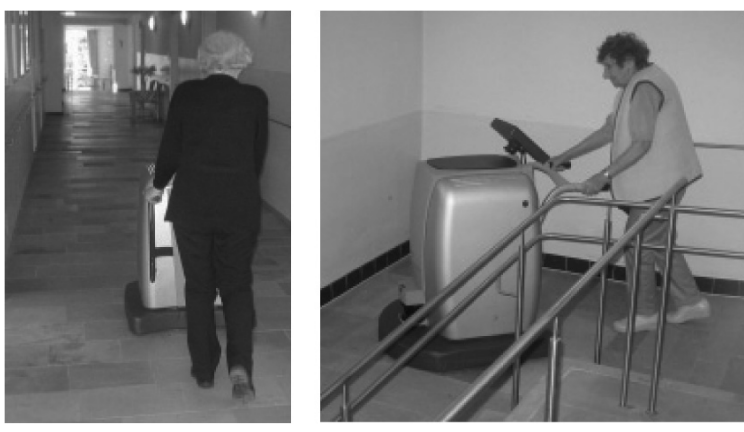

Figure 21. Test persons along the first test path.

target mode. Figure 21 shows two of the test persons on their way between the foyer and the meeting room.

The following results could be obtained from the first test:

- Several test persons had difficulties to control the conventional walker when walking down the ramp as it was rolling away faster than they could follow. This problem did not occur with the robot as the maximum velocity of the robot is constrained and the current velocity of the robot is controlled by the user input alone.

- The time to reach a target was larger with the robot than with the conventional walker. This was caused by the limited maximum velocity of the robot $(0.5 \mathrm{~m} / \mathrm{s})$ compared to the measured maximum velocities of the users with the conventional walker (up to 1.1 $\mathrm{m} / \mathrm{s})$.

- The applied forces to control the robot were significantly higher than with conventional walkers. This was caused by the inertia of the robot's control system, but also because the users were pushing the robot hard trying to exceed its maximum velocity.

- Three of the test persons collided with the environment when using the conventional walker. No collisions occurred using the robotic walker.

- Some persons showed difficulties with the non-holonomic nature of the robot. To rotate the robot on the spot, the user has to move laterally around the robot or turn towards one walking handle. When using the robot in direct control mode, some test persons approached the target in a curve instead of rotating the robot on the spot even though all of them had proven to be able to move laterally.

- One of the test persons did not know how to get to the meeting room and started off in the wrong direction. This happened both with the conventional walker and with the robot in direct control mode (Figure 22, left). In target mode, the user was finally led to the target on the shortest path which reduced the measured walking path by 10 meters compared to the first trial with the conventional walker.

- When moving in target mode, a maximum distance to all obstacles was maintained. Figure 22, right displays how the path was adapted to surround two tables positioned along the wall of the corridor.
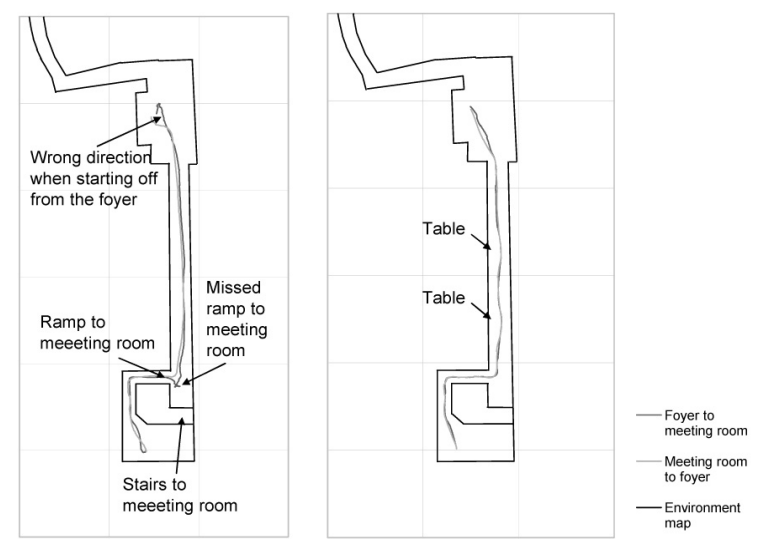

Figure 22. User path in direct control mode (left) and in target mode (right). Grid size: $10 \mathrm{~m}$.

\subsection{Guidance to the First Floor}

The second test was done with the robot in target mode only. A target in the first floor was selected which led the user from the foyer on the ground floor down a curved ramp, to the main entrance hall, and to the entrance door of the cafeteria. The transition between floors was done with an elevator further down the corridor from the cafeteria entrance. In front of the elevator, the robot automatically switched to direct control mode to enable the user to navigate into and out of the elevator. Whereas on the way to the first floor, path modifications by the user were not allowed; direct path modifications and wall following were activated on the way back to the foyer. Figure 23 shows two test persons on the way to the first floor. 

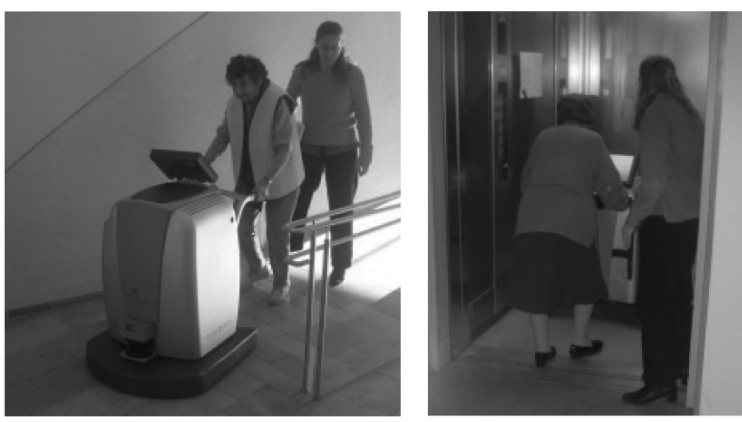

Figure 23. Test persons along the second test path.

The following results could be obtained from the second test:

- All users managed to get in and out of the elevator. However, when moving out of the elevator, controlling the robotic walker in backwards motion seemed slightly more difficult than moving forward. One of the test persons turned the robot inside the elevator to be able to leave it in forward motion.

- As on the path of the second test, a lot more people were walking around, the planned path was modified several times due to persons detected in the way Figure 24 shows a path modification in the entrance hall as the direct way was blocked. All persons could be passed by safely.

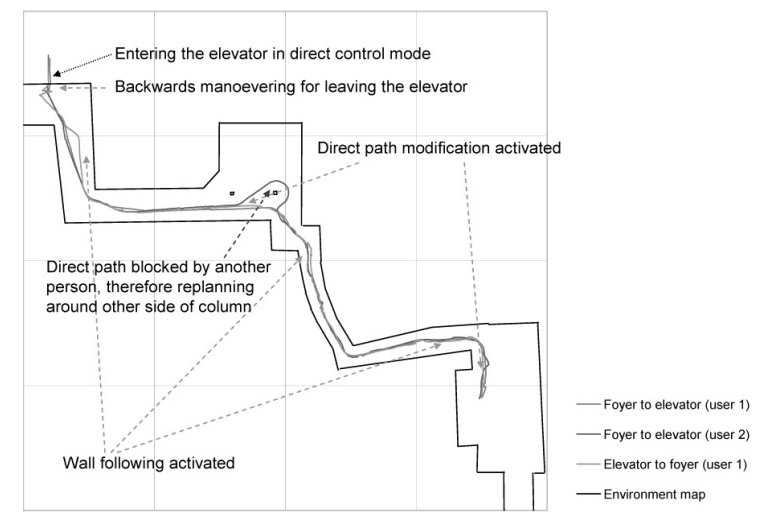

Figure 24. User path between foyer and elevator with and without active user modifications.

- On the way back, path modifications due to user inputs deviating from the planned motion direction were observed frequently. Only $31 \%$ of the time no path modifications were active, direct user modifications were active $27 \%$ of the time, wall following was active $42 \%$ of the time (5). Figure 24 shows the path of the same test person on the way to and from the elevator and indicates the active path modifications on they way from the elevator to the foyer.

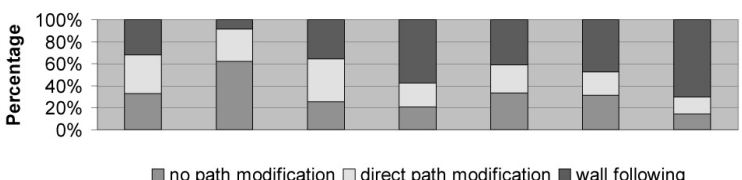

Figure 25. Activation of path modifications.

\section{Conclusion and Outlook}

An adaptive guidance system for robotic walking aids has been presented. The advantage of the guidance system compared to existing guidance systems for robotic walkers is that it maintains the general heading to the target while at the same time considering the input of the user. The support for the user is ensured by a stumble protection module which automatically stops the robot should a stumble motion be detected and by considering the desired motion direction of the user while moving to the target. Field tests in an old people's residence have proven the capabilities of the guidance system. A questionnaire posed to the walker users after the tests showed that $80 \%$ of the users felt safe and in control of the robotic walker. Difficulties occurred due to the limited velocity and the non-holonomic structure of the robot assistant Care-O-bot II. Therefore, future work will concentrate on the development of a new walking aid hardware which enables higher velocities, higher accelerations and thus lower forces to control the robot. The kinematics of the new walker should be similar to conventional walkers, thus the user should be positioned in the turning centre of the robot to enable turning on the spot.

\section{Acknowledgments}

Part of this work has been supported by the MORPHA-project funded by the German Ministry of Education and Research $(\mathrm{bmb}+\mathrm{f})$ under grant 01IL902G/9. 


\section{References}

[1] BÜHLER, Christian; HeCk, Helmut; NedZA, JANUS; WALLBRUCH, RAINER: "Evaluation of the MOBIL Walking \& Lifting Aid". In C. Marincek, Ch. Bühler, H. Knops, R. Andrich (Eds.): Assistive Technology Added Value to the Quality of Life. Amsterdam Berlin Oxford Tokyo Washington/DC: IOS Press, 2001; pp. 210-215.

[2] Charron, P. M. ; Kirby, R. L. And Macleod, D. A.: "Epidemiology of walker-related injuries and deaths in the United States". Amer. J. Phys. Med. Rehab., vol. 74, no. 3, pp. 235-239, 1995.

[3] Chuy JR., Oscar; Hirata, Yasuhisa; Whand, ZHIDONG; KosUGE, KAZUHIRO: "Approach in Assisting a Sit-to-Stand Movement Using Robotic Walking Support System". In: IEEE/RSJ International Conference on Intelligent Robots and Systems, October 2006, Beijing, China, S. 4343-4348.

[4] COOPER, RoRy A.: "Intelligent Control of Power Wheelchairs". In Engineering in Medicine and Biology Magazine, IEEE, Volume: 14, Issue: 4, Jul/Aug 1995, pp. 423-431.

[5] EgAWA, SAKU; TAKEUCHI, IKUO; KOSEKI, ATSUSHI; ISHII, TAKESHI: "Electrically Assisted Walker with Supporter-embedded Force-sensing Device". In Advances in Rehabilitation Robotics: Human-friendly Technologies on Movement Assistance and Restoration for People with Disabilities, Volume 306/2004, Springer Verlag.

[6] Glover, J.; Holstius, D.; ManoJlovich, M.; MONTGOMERY, K.; POWERS, A.; Wu, J.; KIESLER, S.; MATTHEWS, J. AND ThRUN, S.: "A roboticallyaugmented walker for older adults". Technical Report CMU-CS-03-170, Carnegie Mellon University, Comp. Science Dep., Pittsburgh, PA, USA, 2003.

[7] GRAF, B.: „,Reactive Navigation of an Intelligent Robotic Walking Aid“. In Proc. of IEEE International Workshop on Robot and Human Interactive Communication (RoMan), 2001, Paris, France, pp. 353-358.

[8] GRAF, BIRGIT; HANS, MATTHIAS; SCHRAFT, ROLF DIETER: "Care-O-bot II - Development of a Next Generation Robotic Home Assistant". In $\mathrm{Au}$ tonomous Robots 16 (2004), Nr. 2, S. 193-205.

[9] HiRATA, YASUHISA; HARA, ASAMI; MURAKI, ASAMI; KosUGE, KAZUHIRO: "Passive-type Intelligent Walker RT Walker". In Proc. of the 2006 IEEE International Conference on Robotics and Automation, Orlando, Florida, USA, 2006.

[10] Hostalet Wandosell, J. M., Graf, B.: "Nonholonomic Navigation System of a Walking-aid Robot". In Proc. of IEEE International Workshop on Robot and Human Interactive Communication, 2002, Berlin, Germany, pp. 518-523.

[11] Huang, Cunjun; Wasson, Glenn; Alwan, Majd; SHETH, PRADIP; LEDOUX, AlEXANDRE: "Shared Navigational Control and User Intent Detection in an Intelligent Walker". In Proc. of AAAI Fall 2005 Symposium (EMBC), 2005.
[12] Jiang, Kaichun; SEnEVIRATne, LAKMAL, D.; EARLES, S. W. E.: "A Shortest Path Based Path Planning Algorithm for Nonholonomic Mobile Robots". In: Journal of Intelligent and Robotic Systems, 1999, H. 24, pp. 347-366.

[13] Kaye, H. Stephen; Kang, Taewoon; LaPlante, MitCHELl P.: "Mobility Device Use in the United States". Disability Statistics Report, (14), Washington, D.C.: U.S. Department of Education, National Institute on Disability and Rehabilitation Research, 2000 .

[14] LaCEY, Gerard; Dawson-Howe, KenNeth M.: "Personal adaptive mobility aid for frail and elderly blind people". Techn. Rep. TR-CS-95-18, Comp. Science Dept., School of Engineering, Trinity College Dublin, Ireland, 1995.

[15] Latombe, J.-C.: "Robot Motion Planning". $U K$ : Kluwer Academic Publishers, 1996.

[16] LeE, ChOON-Young AND LeE, Ju-JANG: "WalkingSupport Robot System for Walking Rehabilitation: Design and Control". In Artificial Life and Robotics, Volume 4, Number 4 / December, 2000.

[17] LeVINE, Simon P.; Bell, DAVID A.; JAROS, LINCOLN A.; SIMPSON, RICHARD C.; KOREN, YORAM; BORENSTEIN, JOHANN: "The NavChair Assistive Wheelchair Navigation System". In IEEE Trans. on Rehab. Eng., vol. 7, no. 4, 1999.

[18] MÉdÉRIC, P.; PASQUi, V.; Plumet, F.; AND BIDAUd, PH.: "Elderly People Sit-to-Stand Transfer Experimental Analysis". In Proc. of the 8th Int. Conference on Climbing and Walking Robots (CLAWAR 2005), pp. 953-960.

[19] MORRIS, A.; DONAMUKKala, R.; KAPURIA, A.; STEINFELD, A.; MATTHEWS, J.; DUNBAR-JACOBS, J. AND THRUN, S.. "A robotic walker that provides guidance". In Proc. of the IEEE International Conference on Robotics and Automation (ICRA), Taipei, Taiwan, 2003.

[20] NejatBakhsh, N.; Kosuge, K.: "User-Environment Based Navigation Algorithm for an Omnidirectional Passive Walking Aid System". In Proc. of the 9th International Conf. on Rehab. Robotics, Chicago, USA, 2005.

[21] Prassler, ERWIN; SchOlZ, JENS AND FIORINI, PAOLO: "A Robotic Wheelchair for Crowded Public Environments". In IEEE Robotics and Automation Magazine 8(1): 38-45.

[22] Quinlan, Sean; Khatib, OUSSAma: "Elastics bands: Connecting Path Planning and Control". In IEEE Transactions on Robotics and Automation, 1993.

[23] Rodríguez-LosadA, Diego; Matía, Fernando; JimÉnEZ, Agustín; Galán, RAMÓn AND LACEY, GERARD: "Guido, the Robotic Smart Walker for the frail visually impaired". First International Congress on Domotics, Robotics and Remote Assistance for All - DRT4all 2005. Act Book. ISBN: 84-88934-22-X. pp. 155-169. Madrid, Spain, 2005. 
[24] Sabatini, A. M.; Genovese, V.; PaCchierotti, E., "A Mobility Aid for the Support to Walking and Object Transportation of People with Motor Impairments". Proc. of IEEE/RSJ Intl. Conf. on Int. Robots and Systems, 2002.

[25] ShIM, HyeOn-MiN; LeE, EunG-HyuK; ShIM, JAEHong; LeE, SANG-MoO; Hong, SEung-Hong: "Implementation of an Intelligent Walking Assistant Robot for the Elderly in Outdoor Environment". In Proc. of the 9th International Conf. on Rehab. Robotics, Chicago, USA, 2005.

[26] Stat. Bundesamt Deutschland: "11. koordinierte Bevölkerungsvorausberechnung", www .destatis.de, 2006.

[27] YANCO, Holly A.: "Shared User-computer Control of a Robotic Wheelchair System". PhD Thesis at the Massachusetts Institute of Technology, September 2000.

[28] Yu, Haoyong; Spenko, Matthew; Dubowsky, STEVEN: "An Adaptive Shared Control System for an Intelligent Mobility Aid for the Elderly". In Autonomous Robots 16 (2003), Nr. 15, S. 53-66.

Received: November, 2007 Accepted: March, 2008

Contact address: Birgit Graf Fraunhofer IPA Nobelstr. 12 70569 Stuttgart, Germany e-mail: birgit.graf@ipa.fraunhofer.de www. care-o-bot.de

BIRGIT GRAF is a group manager at the Fraunhofer Institute for Manufacturing Engineering and Automation (IPA) in Stuttgart, Germany. The research field of her group is the development of new systems and technologies for domestic service robots and personal robots. 